\title{
Towards a framework for treatment effectiveness in schizophrenia
}

\author{
This article was published in the following Dove Press journal: \\ Neuropsychiatric Disease and Treatment \\ 24 September 2014 \\ Number of times this article has been viewed
}

\author{
Georg Juckel' \\ Andrea de Bartolomeis ${ }^{2}$ \\ Philip Gorwood ${ }^{3}$ \\ Sergey Mosolov ${ }^{4}$ \\ Luca Pani ${ }^{5}$ \\ Alessandro Rossi ${ }^{6}$ \\ Julio Sanjuan ${ }^{7}$ \\ 'Department of Psychiatry, LWL- \\ University Hospital, Ruhr-University \\ Bochum, Bochum, Germany; \\ ${ }^{2}$ Laboratory of Molecular Psychiatry \\ and Unit of Treatment Resistant \\ Psychosis, University School of \\ Medicine of Naples Federico II, \\ Napoli, Italy; ${ }^{3}$ Groupe Hospitalier \\ Sainte-Anne (CMME), Paris-Descartes \\ University, Paris, France; ${ }^{4}$ Moscow \\ Research Institute of Psychiatry, \\ Moscow, Russia; Institute of \\ Translational Pharmacology, Italian \\ National Research Council, Rome, \\ Italy; 'Università de L'Aquila, L'Aquila, \\ Italy; ${ }^{7}$ Clinic Hospital, Spanish Mental \\ Health Network (CIBERSAM), \\ University of Valencia, Valencia, Spain
}

Correspondence: Georg Juckel Department of Psychiatry, LWLUniversity Hospital, Ruhr-University Bochum, Bochum 4479I, Germany

Tel +492345077 I I 00

Fax +49 2345077 I I 9

Email g.juckel@wkp-Iwl.org
Introduction: Prompt administration of antipsychotic treatment that is adhered to is essential for the optimal treatment of schizophrenia. Many patients have benefited from the advent of second-generation antipsychotics, which can offer good symptomatic control with reduced incidence of extrapyramidal symptoms, although with higher risk of metabolic side effects. It is unsurprising that accounts as to whether first- and second-generation antipsychotics differ in their efficacy vary, since treatment effectiveness is a broad notion and difficult to define.

Objectives: Numerous factors may be used to gauge treatment effectiveness and, while it has largely been defined in terms of improvements in four domains (symptoms of disease, treatment burden, disease burden, and health and wellness), the real-world clinical utility of this consensus is unclear. Therefore, this article aims to provide a framework that can aid psychiatrists in making assessments about treatment effectiveness.

Methods and results: A panel of 12 psychiatrists and psychopharmacologists convened to develop and propose an accessible and globally-applicable framework for assessing the effectiveness of antipsychotic treatments in patients with schizophrenia. Following presentation of a preliminary proposal to a wider group of psychiatrists from across Europe, it was refined into a framework comprising five domains: symptomatic remission and retention of treatment; affective symptoms; cognitive functioning; treatment satisfaction; and personal and social functioning - each of which is discussed in this article.

Conclusions: This article provides a framework that can aid psychiatrists in making assessments about treatment effectiveness. It is anticipated that the framework outlined here may contribute to improving clinical practice through the promotion of a patient-centered approach to the assessment of treatment effectiveness, using five specified domains, in patients with schizophrenia.

Keywords: antipsychotic, assessment scales, functioning, mental illness, satisfaction

\section{Introduction}

Prompt administration of effective antipsychotic treatment that is adhered to by patients is important in schizophrenia in order to prolong periods of good symptom control and reduce the risk of relapse. ${ }^{1-3}$ Many patients have benefited from the availability of second-generation antipsychotics (SGAs), which provide improved overall efficacy with reduced incidence of extrapyramidal symptoms compared with first-generation antipsychotics. ${ }^{2,4}$ Conversely, some SGAs are associated with a relatively greater risk of weight gain and metabolic side effects. ${ }^{5-7}$ There are significant variations in the receptor-binding profiles of SGAs that contribute to their differing efficacy across many of the symptomatic domains of schizophrenia ${ }^{8}$ as well as to differences in their tolerability. ${ }^{9}$ Meta-analyses of the efficacy of antipsychotics have distinguished only small differences between individual antipsychotics in terms of alleviating symptoms of the disease. ${ }^{6,7,10}$ However, large-scale naturalistic effectiveness studies such as the 
European First Episode Schizophrenia Trial (EUFEST) and Clinical Antipsychotic Trials of Intervention Effectiveness (CATIE) have demonstrated significant differences in allcause discontinuation between antipsychotics. ${ }^{11,12}$

In clinical practice, psychiatrists are faced with the challenge of selecting the most appropriate treatment strategy for their patient. Since effectiveness is a broad holistic notion, it is difficult to define precisely; consequently, numerous factors can be used to gauge treatment effectiveness over the short and long term. Nevertheless, psychiatrists must consider the relevance of each of these factors in assessing effectiveness in specific patient populations, since those factors considered appropriate for patients during the acute phase $^{13}$ may be less suitable when applied to stable or chronic patients. ${ }^{14}$ A solution to clinical heterogeneity is to "stage" the longitudinal trajectories in order to optimize treatment. ${ }^{15}$

A variety of indirect measures of effectiveness has been used in recent naturalistic observational studies (Table 1). Treatment retention, or time to discontinuation, is one of the more commonly used measures of effectiveness in largescale studies, such as CATIE and EUFEST, ${ }^{11,12,16}$ where it is necessary to include a single quantifiable endpoint for the duration of the study. However, psychiatrists should aim to change an ineffective therapy proactively (for example, due to insufficient/inadequate symptom control or lack of patient acceptance) for one they believe will be more effective, before treatment discontinuation. Thus, treatment discontinuation as a measure of effectiveness is of limited value in clinical practice. As such, a more comprehensive and continuous measure, encompassing numerous domains that can be observed throughout the treatment course, is needed to help

Table I Primary endpoints from large pragmatic studies

\begin{tabular}{|c|c|c|}
\hline Trial & Primary endpoint & References \\
\hline $\mathrm{SOHO}$ & $\begin{array}{l}\text { CGI, quality of life, social } \\
\text { functioning }\end{array}$ & $\begin{array}{l}\text { Dossenbach et al }{ }^{17} \\
\text { Haro et al }\end{array}$ \\
\hline CUtLASS & Quality of life & $\begin{array}{l}\text { Jones et } \mathrm{a}^{19} \\
\text { Lewis et } \mathrm{a}^{20}\end{array}$ \\
\hline EUFEST & $\begin{array}{l}\text { Loss of retention (time to } \\
\text { discontinuation) }\end{array}$ & Kahn et al" \\
\hline BETA & CGI improvement & Tandon et $\mathrm{al}^{21}$ \\
\hline CATIE & All cause discontinuation & $\begin{array}{l}\text { Lieberman et al } \\
\text { Stroup et } \text { al }^{16}\end{array}$ \\
\hline CAFE & All cause discontinuation & McEvoy et $\mathrm{a}^{22}$ \\
\hline
\end{tabular}

Abbreviations: BETA, Broad Effectiveness Trial with Aripiprazole; CAFE, Comparison of Atypicals in First Episode Psychosis; CATIE, Clinical Antipsychotic Trials of Intervention Effectiveness; CGI, Clinical Global Impression; CUtLASS, Cost Utility of the Latest Antipsychotic Drugs in Schizophrenia Study; EUFEST, European First Episode Schizophrenia Trial; SOHO, Schizophrenia Outpatient Health Outcome. identify issues that may lead to relapse or discontinuation prior to their occurrence. Measures of effectiveness used in these trials also fail to account for the factors that patients often use to describe how effective they believe their treatment to be, such as more energy and motivation for personal and occupational activities. Furthermore, as patients respond to therapy and become capable of taking on more responsibilities in their daily lives, additional stressors may lead to a reemergence of symptoms and apparent ineffectiveness of their treatment. Thus, in order to refine the assessment of treatment effectiveness in everyday clinical practice, a patient-centered approach focused on real-world outcomes should be considered.

Clinical studies that have investigated predictors of treatment discontinuation are informative, given the importance of treatment retention and the need to take steps to avoid discontinuation before it happens. In a study of firstepisode patients by Perkins et $\mathrm{al}^{23}$ poor treatment response $(P<0.001)$ and low medication adherence $(P=0.02)$ were independent predictors of discontinuation against medical advice. Ongoing substance abuse, ongoing depression, and treatment response failure significantly predicted poor medication adherence $(P<0.01)$. Higher cognitive performance at baseline was also associated with lower medication adherence $(P<0.05)$. Therefore, it is crucial to try to identify such modifiable predictors when present and address them where possible.

Guidance for practicing psychiatrists regarding the assessment of treatment effectiveness in their patients has been somewhat limited thus far. Effectiveness has been defined as improvements in four domains: symptoms of disease (measured using symptom scales); treatment burden (measured using adverse event scales); disease burden (assessed by interview with patients and families); and health and wellness (measured using quality-of-life scales). However, the real-world clinical utility of this consensus is unclear. $^{24}$

In an attempt to provide a framework that can inform psychiatrists of the effectiveness of a particular treatment in their patients, a panel of European experts convened to discuss and develop a consensus on key measures of effectiveness that should be monitored in patients with schizophrenia receiving antipsychotic treatment. This manuscript summarizes these measures and explains the rationale for their inclusion. The aim was not to propose a formally validated guideline, but rather to provide a framework that can inform psychiatrists of the effectiveness of a particular treatment in their patients and assist them in their everyday clinical practice. 


\section{Development of the framework for assessing treatment effectiveness}

There is currently a need for a concise and pragmatic framework with which to assess antipsychotic effectiveness. To address this unmet need, a panel of 12 European psychiatrists and psychopharmacologists (including the authors of this paper), with extensive experience of treating patients with schizophrenia and an interest in aspects of treatment effectiveness, convened to propose a framework for assessing the effectiveness of antipsychotic treatments in patients with schizophrenia. As well as considering the available clinical evidence, the panel discussed domains that could potentially be used to assess effectiveness in clinical practice. In addition to symptomatic remission and retention in treatment, ten domains were initially proposed. The panel also discussed available methodologies for the measurement of effectiveness of antipsychotic medication in patients with schizophrenia. Scales for measuring effectiveness were then selected for inclusion in the framework on the basis of their specificity for the domain, clinical relevance, and ease of use for everyday clinical practice. The merits of each of the constituent domains and associated scales were further discussed and refined by the panel. The five most relevant domains were included in the framework of treatment effectiveness in patients with schizophrenia. Subsequently, this framework was presented at a forum of 149 European psychiatrists involved in the everyday treatment and management of patients with schizophrenia. Each attendee rated the domains in terms of their importance in defining effectiveness, and rated the proposed scales for their usefulness in assessing effectiveness in clinical practice. Based on the feedback from attendees, the constituent domains of the framework and the recommended scales by which to assess them were finalized to generate an easy-to-use and globally-applicable framework of treatment effectiveness. This initiative, including all the clinician meetings at which the domains were developed and refined, was supported by Janssen. The rationale for inclusion of each of these five domains is discussed here along with supporting literature and the resultant recommendations for the framework.

\section{Use of this framework}

This framework has been proposed, based on the subjective opinions of the panel members, in an attempt to define better how to assess the effectiveness of antipsychotic medications in the treatment of schizophrenia, with a view to meeting the individual needs of psychiatrists and patients. Although the panel recognizes the need for an integrated treatment approach for patients, this framework was developed with a focus on treatment with antipsychotic medication. Therefore, the use of these proposals for other available treatments, such as psychosocial therapies, is at the discretion of the physician. The framework of treatment effectiveness should be applied to patients who continue to attend appointments and who have ongoing symptoms requiring treatment. The framework of effectiveness presented here should not be seen as an empirical tool or training guide. Furthermore, although the panel encourages an examination of all domains and a holistic approach to care, the data obtained from individual domains should not be summed to give a single numerical measure of effectiveness. To gain greater insight into the impact of a given treatment strategy on the everyday lives of their patients, physicians should aim to increase the involvement of their patient's family or caregiver in the assessment of treatment effectiveness.

\section{Domains of the framework of treatment effectiveness for antipsychotic therapy in patients with schizophrenia}

Therapeutic efficacy, defined as symptomatic remission ${ }^{25}$ and treatment retention, was selected as the core domain of the framework of treatment effectiveness, since it is a readily acknowledged and understood measure. However, the panel considered that, in order to assess the effectiveness of an antipsychotic therapy successfully, it is important to move beyond the common assessments of efficacy and include domains that may be overlooked in clinical practice. As such, the panel proposed and discussed ten domains that, in addition to symptomatic remission and retention in treatment, could be used to assess effectiveness in clinical practice:
1. Cognitive functioning
2. Personal and social functioning
3. Engagement in treatment
4. Treatment satisfaction
5. Quality of life
6. Affective symptoms
7. Well-being
8. Hospitalization
9. Relapse
10. Tolerability.

Domains were selected for inclusion in the final framework if, based on the opinions and experience of the panel, they were clinically applicable, clearly defined, and measurable; additionally, some factors were also excluded if they were considered to represent a component of, or contribute 
significantly to, other suggested domains. For example, the group believed tolerability to medication was adequately represented by treatment retention and treatment satisfaction, since patients would discontinue or report dissatisfaction with treatment associated with unacceptable side effects if their views were elicited. Relapse and hospitalization were considered for inclusion but ultimately excluded on the basis that they are both measures that occur too late in the treatment course. The framework was intended to enable the effectiveness of treatment to be monitored prior to relapse or hospitalization. For the final framework of treatment effectiveness in patients with schizophrenia, five domains were included:

1. Symptomatic remission and treatment retention

2. Affective symptoms

3. Cognitive functioning

4. Treatment satisfaction

5. Personal and social functioning.

The five domains were then presented to the wider group of 149 European psychiatrists who were asked to rate the inclusion of each domain as "essential", "very important", "desirable", or "irrelevant" to the framework of effectiveness in schizophrenia (Table 2).

Having received feedback and endorsement from the wider group of European psychiatrists on the domains to be included in the framework of effectiveness, the panel considered the interrelationship of the domains to one another. The domains do not necessarily contribute equally or operate independently of one another (Figure 1). For example, it could logically be assumed that retention in treatment may influence symptomatic remission, cognitive functioning, and affective symptoms. Any improvement in each or all of these may benefit personal and social functioning, whereas greater treatment satisfaction may follow with prolonged retention in treatment.

\section{Symptomatic remission and retention in treatment}

The primary aim of antipsychotic treatment is to reduce positive and negative symptoms, and maintain periods of symptom control., ${ }^{2,3}$ There is an increasing body of literature that demonstrates that early efficacy of antipsychotic therapy is one of the few consistent predictors of later efficacy in patients with first-episode psychosis, ${ }^{26}$ as well as in patients with acute ${ }^{27}$ and chronic ${ }^{25,28}$ schizophrenia. Inadequate symptom control has also been reported to be the most common reason for early treatment discontinuation. ${ }^{29}$ Therefore, it is essential that symptomatic remission and retention of treatment forms the core domain of the framework of treatment effectiveness. There are numerous tools that have been developed to measure symptoms of schizophrenia and, therefore, determine symptom remission, such as the positive and negative syndrome scale (PANSS), and the brief psychiatric rating scale. However, the suitability of these scales for clinical practice, as opposed to a clinical trial setting, must be considered. For instance, clinic appointments are often time-restricted; consequently, lengthy assessments are not possible. Furthermore, although many of the scales could be used to assess symptoms adequately, most do not sufficiently capture all of the relevant factors of symptomatic remission, including positive and negative symptoms and their duration. Thus, the panel considered the criteria proposed by Andreasen et $\mathrm{al}^{30}$ for symptomatic remission, which utilizes PANSS, in order to provide greater clarity around treatment goals, as well as an improved framework for the design and comparison of investigational trials. In one study, the majority of patients with assured compliance with antipsychotic medication following a first-psychotic episode, in the form of long-acting injectable risperidone, achieved sustained remission, as defined by the Andreasen criteria, and maintained this for the length of the study. ${ }^{31}$ Treatment with the long-acting injectable formulations of both olanzapine ${ }^{32}$ and aripiprazole ${ }^{33}$ have also been associated with significant improvements in PANSS total scores compared with placebo. Moreover, some trials have shown differences in the proportion of chronic patient populations achieving remission, as defined by the Andreasen criteria, with different antipsychotics. For example, in a 196-week study, patients treated with ziprasidone (80-160 mg/day given twice daily, or $80-120 \mathrm{mg} /$ day given once daily) were

Table 2 Importance of the framework domains as voted for by 149 psychiatrists attending a large educational meeting

\begin{tabular}{lllll}
\hline Domain & Essential (\%) & Very important (\%) & Desirable (\%) & Irrelevant (\%) \\
\hline Symptomatic remission & 28 & 51 & 19 & 2 \\
Retention in treatment & 40 & 46 & 11 & 3 \\
Affective symptoms & 16 & 73 & 9 & 2 \\
Cognitive functioning & 14 & 56 & 21 & 9 \\
Treatment satisfaction & 43 & 40 & 9 & 8 \\
Personal and social functioning & 43 & 40 & 14 & 3 \\
\hline
\end{tabular}




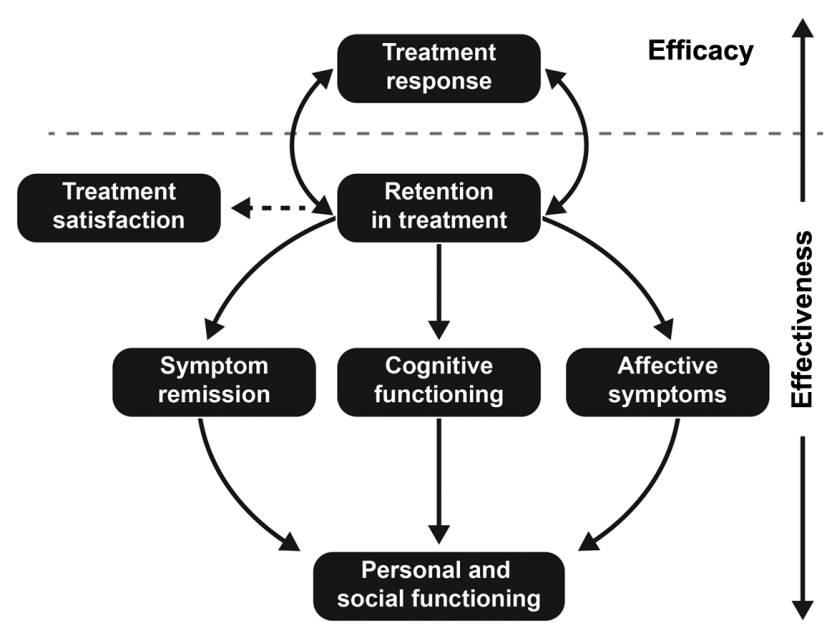

Figure I The hypothesized interaction between effectiveness domains.

more likely to achieve remission (51\%) compared with those treated with haloperidol (5-20 mg/day) $(40 \% ; P=0.04){ }^{34}$

\section{Recommendation for symptom remission}

The remission criteria proposed by Andreasen et $a l^{30}$ focuses on psychosis, defining treatment response, and having a set timeframe. The increasing use of these criteria, which is reflected in the volume of literature, indicates that they are an important and helpful tool in the field of psychiatry. ${ }^{35}$

A score of $\leq 3$ (mild) for 6 consecutive months for all eight PANSS items (using the 1-7 range for each PANSS item) is representative of remission as proposed in the remission criteria from Andreasen et al. ${ }^{30}$ The individual PANSS items comprise (with the specific PANSS items shown in parentheses):

- Delusions (P1)

- Unusual thought content (G9)

- Hallucinatory behavior (P3)

- Conceptual disorganization (P2)

- Mannerisms/posturing (G5)

- Blunted affect (N1)

- Social withdrawal (N4)

- Lack of spontaneity (N6).

Although there is a lack of consensus in the literature as to how often assessment of symptomatic remission should be conducted, ${ }^{36,37}$ the panel believed this should be undertaken monthly.

Ensuring that patients continue to take their medication is an important issue for any physician treating patients with a chronic disease. The measurement of treatment retention in patients with schizophrenia can be challenging since it relies heavily on patients or their families informing their psychiatrist that they have discontinued treatment. In addition, physicians have to consider exactly which time scale and conditions differentiate treatment retention from treatment discontinuation.

\section{Recommendation for treatment retention}

The panel proposed that, as a practical measure, treatment retention should be considered over a period of approximately 12 months. Assessment by the treating psychiatrist and nursing staff should be maintained during regular sessions with patients; during the sessions, patients should be asked about their medication-taking behavior. Furthermore, any gaps in treatment should be recognized and considered in relation to the overall effectiveness of treatment according to this framework. The panel discussed and agreed that, based on their clinical experience, gaps in therapy of $<4$ weeks on less than four occasions in any 12-month period were considered acceptable for treatment retention.

\section{Affective symptoms}

The alleviation of affective symptoms, such as depression and anxiety, is recognized as one of the unmet clinical needs of schizophrenia. However, when assessing treatment effectiveness, many physicians may not always consider their impact on affective symptoms; therefore, there is need for increased clinical awareness regarding their routine measurement. Many scales can be used to assess affective symptoms, such as the Hamilton anxiety rating scale, ${ }^{38,39}$ Montgomery-Åsberg Depression Rating Scale, ${ }^{40}$ PANSS subscale items, and Calgary Depression Scale for Schizophrenia. ${ }^{41}$ Clinical data regarding the effect of treatment on affective symptoms are sparse compared with the available data on positive symptoms. However, some recent trials have demonstrated a positive correlation between treatment with SGAs and improved affective symptoms. ${ }^{42-44}$ In a study of 60 outpatients with chronic schizophrenia, there was no difference between amisulpride and olanzapine with regard to positive, negative, and cognitive symptoms. Nevertheless, patients treated with olanzapine had significantly lower scores on the PANSS subscale items for depression/ anxiety compared with amisulpride $(P<0.05){ }^{42}$ Treatment with paliperidone extended release also improved PANSS subscale scores for anxiety and depression compared with placebo $(P<0.001)$; however, this trial was not sufficiently powered to draw conclusions based on individual PANSS factors. ${ }^{44}$ Improvements in PANSS depression cluster scores (PANSS subscale items G1 [somatic concern], G2 [anxiety], G3 [guilt feelings], and G6 [depression]) were observed in patients receiving quetiapine XR (600-800 mg), compared 
with placebo (separation from placebo based on 95\% confidence interval). ${ }^{43}$

\section{Recommendations for affective symptoms}

The panel believed that treatment of affective symptoms in patients with schizophrenia should be given higher importance by physicians since improvements can bring significant improvement to patient quality of life. Assessment of affective symptoms should be performed monthly.

To assess affective symptoms, PANSS subscale items were selected since they are easy to use and are applicable in clinical practice. The panel considered a rating better than 'mild' ( $\leq 3$ ) for anxiety (G2) and depression (G6) was important when considering the impact of affective symptoms.

The use of the Calgary Depression Rating Scale may also be relevant, when a more detailed analysis of patient depression is required, since this assessment scale is based largely on patient questionnaires; however, the Calgary Depression Rating Scale does not cover anxiety so it cannot be used for assessment of all affective symptoms.

\section{Cognitive functioning}

Antipsychotics are known to be efficacious for the treatment of many of the symptoms associated with schizophrenia. However, few studies have demonstrated an effect of antipsychotics on the cognitive deficits associated with schizophrenia. In the CATIE study, a small but statistically significant improvement from baseline in neurocognition was observed after 2 months of antipsychotic treatment using olanzapine $(P<0.002)$, perphenazine $(P<0.001)$, quetiapine $(P<0.001)$, risperidone $(P<0.001)$, and, to a lesser extent, ziprasidone $(P<0.06) .{ }^{45}$ At present, there is an absence of compelling data to indicate that SGAs are more effective than first-generation antipsychotics in ameliorating cognitive symptoms and, without a proven effective treatment, cognitive functioning is often overlooked in clinical practice. This is further compounded by a lack of training and experience regarding the assessment of cognitive functioning, and the misconception that cognitive functioning is only of theoretical, rather than clinical, importance. Additionally, the available tools for assessment are largely inadequate in determining the efficacy of a treatment on cognitive functioning. ${ }^{46}$ Symptom scales are not appropriate since data from EUFEST suggested that cognitive measures, including processing speed, motor dexterity, verbal memory, and cognitive flexibility, appear to be only weakly correlated with psychopathological dimensions. ${ }^{47}$

The panel discussed a wide range of assessment scales that could be used for cognitive functioning, considering factors such as ease of use, time required to conduct them, training of personnel required, and clinical applicability. For instance, the short form of the University of California, San Diego, Performance-Based Skills Assessment (UPSA-B) ${ }^{48}$ was considered a reliable scale, particularly for the assessment of patients prior to discharge from hospital, but was viewed as too cumbersome for routine use in the clinic. Most discussion focused on the National Institute of Mental Health's Measurement and Treatment Research to Improve Cognition in Schizophrenia (MATRICS) Consensus Cognitive Battery ${ }^{49}$ and the Brief Assessment of Cognition in Schizophrenia scale. ${ }^{50}$

\section{Recommendation for cognitive function}

The assessment of cognitive function should be performed using the simplest tool available. The majority of scales that assess cognitive functioning are applicable only to clinical trials and are unsuitable for routine clinical practice. The Brief Assessment of Cognition in Schizophrenia scale ${ }^{50}$ was recommended as the most practicable scale for everyday use as it can be administered in less than 30 minutes with minimal extra time for scoring and minimal training demands. The panel recommended that cognitive functioning should be assessed annually.

\section{Treatment satisfaction}

Treatment satisfaction is a heterogeneous concept, and its influence on the assessment of effectiveness may only be marginal; however, a framework of effectiveness that fails to include an element of the patient's own view (ie, satisfaction with treatment) would be incomplete. Many physicians associate treatment satisfaction with the efficacy and tolerability of a patient's medication; nevertheless, many patients receive nonpharmacological interventions, such as psychosocial therapies, ${ }^{51}$ which may also influence treatment satisfaction and should be considered in the context of effectiveness. Moreover, antipsychotics produce some complex subjective effects beyond the reduction of clinical symptoms. ${ }^{42}$ There are numerous scales and questionnaires that can be used to assess a patient's satisfaction with their treatment, including the Client Satisfaction Questionnaire, ${ }^{52}$ Verona Service Satisfaction Scale,${ }^{53}$ Client Assessment of Treatment, ${ }^{54}$ Quality of Life Enjoyment and Satisfaction Questionnaire, ${ }^{55}$ Treatment Satisfaction Questionnaire for Medication (TSQM), ${ }^{56}$ and the Medication Satisfaction Questionnaire (MSQ). ${ }^{57,58}$

\section{Recommendation for treatment satisfaction}

The panel agreed that the $\mathrm{MSQ}^{57}$ is an appropriate tool to measure treatment satisfaction since it is quick and simple to 
use and appears to be sensitive to treatment effects. The panel discussed the frequency of assessing treatment satisfaction and, based on their experience, considered monthly assessment of treatment satisfaction to be satisfactory.

- The MSQ is a one-item ("Are you satisfied with your treatment?') global patient-rated seven-point scale, ranging from extremely dissatisfied (1) to extremely satisfied (7).

\section{Personal and social functioning}

The effectiveness of a medication is often described in terms of its efficacy; for example, the effect of medication on positive symptoms is often rapid and relatively easily assessed. However, long-term effects of medication on social functioning and negative symptoms are now also considered essential to improving the quality of life in patients with schizophrenia. ${ }^{59}$ The EUFEST demonstrated improved Global Assessment of Functioning (GAF) scale scores from baseline with significant $(P=0.006)$ differences between the antipsychotic treatment groups in first-episode patients. ${ }^{11}$ Further analysis of this study demonstrated that persistent negative symptoms were associated with a poor response to treatment and worse global functioning after 1 year of antipsychotic treatment. ${ }^{60}$ Social functioning in schizophrenia should be considered an integral component of the framework of effectiveness. Unfortunately, the routine formal assessment of social functioning in psychiatry is not optimal since psychiatrists' assessment of social functioning is often determined subjectively during the clinical interview rather than in a systematic or consistent manner. This may be due to the difficulties associated with measuring social functioning by comparison with the relative ease of measuring symptomatology. The assessment of social functioning is essentially normative and can vary according to context and expectations. The definition of social functioning is also unclear, with a range of overlapping concepts frequently used, such as social disability, social exclusion/ inclusion, quality of life, and needs assessments. Moreover, psychiatrists are not in agreement on what constitutes a direct measure, indirect measure, or risk factor for social functioning impairments. ${ }^{61}$ As a consequence, there is little consensus on the most appropriate method of measurement.

The panel discussed four scales for the measurement of personal and social functioning: the GAF scale; the Social and Occupational Functional Assessment Scale (SOFAS); the Social Inclusion Index scale; and the Personal and Social Performance (PSP) scale. More recently developed instruments, such as the Functional Remission of General Schizophrenia scale, had not been fully validated when producing the framework of treatment effectiveness and were, therefore, excluded from consideration by the panel. Clinically meaningful improvements in personal and social functioning have been shown in patients with different clinical features and courses of schizophrenia when achieving remission under the SGA treatment. ${ }^{62-64}$ Furthermore, EUFEST demonstrated significant differences in functioning of first-episode patients over time between treatment groups. ${ }^{11}$ Following discussion, the panel also excluded the GAF, SOFAS, and Social Inclusion Index scales based on clinical experience of their sensitivity and specificity in measuring personal and social functioning.

\section{Recommendations for personal and social functioning}

Formal assessment of psychosocial functioning should form a greater part of current psychiatric practice and, ideally, be measured by direct contact with the patient or be based on the report of a health care professional that, through longstanding/ ongoing consultation, is sufficiently familiar with the patient. Verbal reports are largely used for the assessment of social skills; however, there is a large discrepancy between what patients say and what they actually do, as well as between the reports provided by patients and those of their caregivers or relatives. ${ }^{17,65}$ If possible, personal and social functioning should be assessed on a monthly basis. The panel believes that the PSP scale ${ }^{59,66}$ is the most appropriate scale to measure personal and social functioning in patients with schizophrenia in clinical practice.

The PSP scale is:

- A 100-point scale assessing four domains:

1. Socially useful activities

2. Personal and social relationships

3. Self care

4. Disturbing and aggressive behaviors

- Clinically validated in numerous countries and across different patients ${ }^{67-72}$

- Independent of symptomatic improvements with regard to outcomes

- Quick to perform.

Psychotic symptoms are known to influence the level of functioning in patients with schizophrenia, yet it has been argued that symptoms and functioning should be measured separately as improvement in one measure does not guarantee improvement in the other. ${ }^{73}$ Most clinical trials assess symptom control and functioning in parallel. The GAF scale has been criticized for including symptoms as part of the total score, thus influencing the measurement of true functioning. ${ }^{59}$ The PSP scale was developed in order to overcome this limitation ${ }^{67}$ and has been validated in at least six languages. ${ }^{67,70,71,74-76}$ However, 
it is well known that psychopathology, as measured with the PANSS, also has some impact on the PSP rating scale. In a study evaluating the reliability and validity of the PSP scale in measuring functioning, the scale was found to be moderately sensitive to illness severity. ${ }^{68}$ In particular, a significant negative correlation has been observed between the negative-symptom subscale of PANSS and the PSP total score. ${ }^{75}$ It is speculated that the negative symptoms of schizophrenia have a greater impact on social functioning than positive symptomatology, as they are likely to result in a greater decline in daily activities (eg, if a person suffers from loss of motivation, social functioning can be especially restricted). There is also a broad debate on the effect of psychopathology, particularly positive symptoms, on the PSP subdimension of "disturbing and aggressive behavior" ${ }^{77}$ This subdimension was added following reliability studies due to the substantial impact of aggressive behavior on patients. ${ }^{67}$ However, it has been suggested that this PSP subdimension is mainly related to lack of judgment and insight rather than the strength of positive symptoms, per se. ${ }^{78,79}$

\section{Proposed framework}

The proposals for the framework with which to assess treatment effectiveness in patients with schizophrenia are summarized in Figure 2. Importantly, for clinical practice, all of the assessments proposed can be performed in approximately 1 hour (Table 3 ).

\section{Limitations}

This proposal for a framework for the assessment of treatment effectiveness in patients with schizophrenia was developed based on the panels' own professional but subjective experiences and opinions. A systematic literature review or clinical research for the express purpose of developing this framework was not performed. As such, the extent of the panel's collective experience with regard to treatment effectiveness is an acknowledged limitation of this work. The recommendations for the monthly assessments discussed in this manuscript are based upon the panel's development of an ideal framework. However, the panel recognizes that, during periods of remission or when it is not possible to make monthly assessments, evaluations may be less frequent. Furthermore, this framework has not been empirically tested regarding feasibility in real-world clinical settings. The potential impact on clinical outcomes is, therefore, unknown. However, the recommendations are intended to assist psychiatrists in their everyday clinical practice, to help inform them on the effectiveness of a particular treatment in their patients.

\section{Effectiveness}

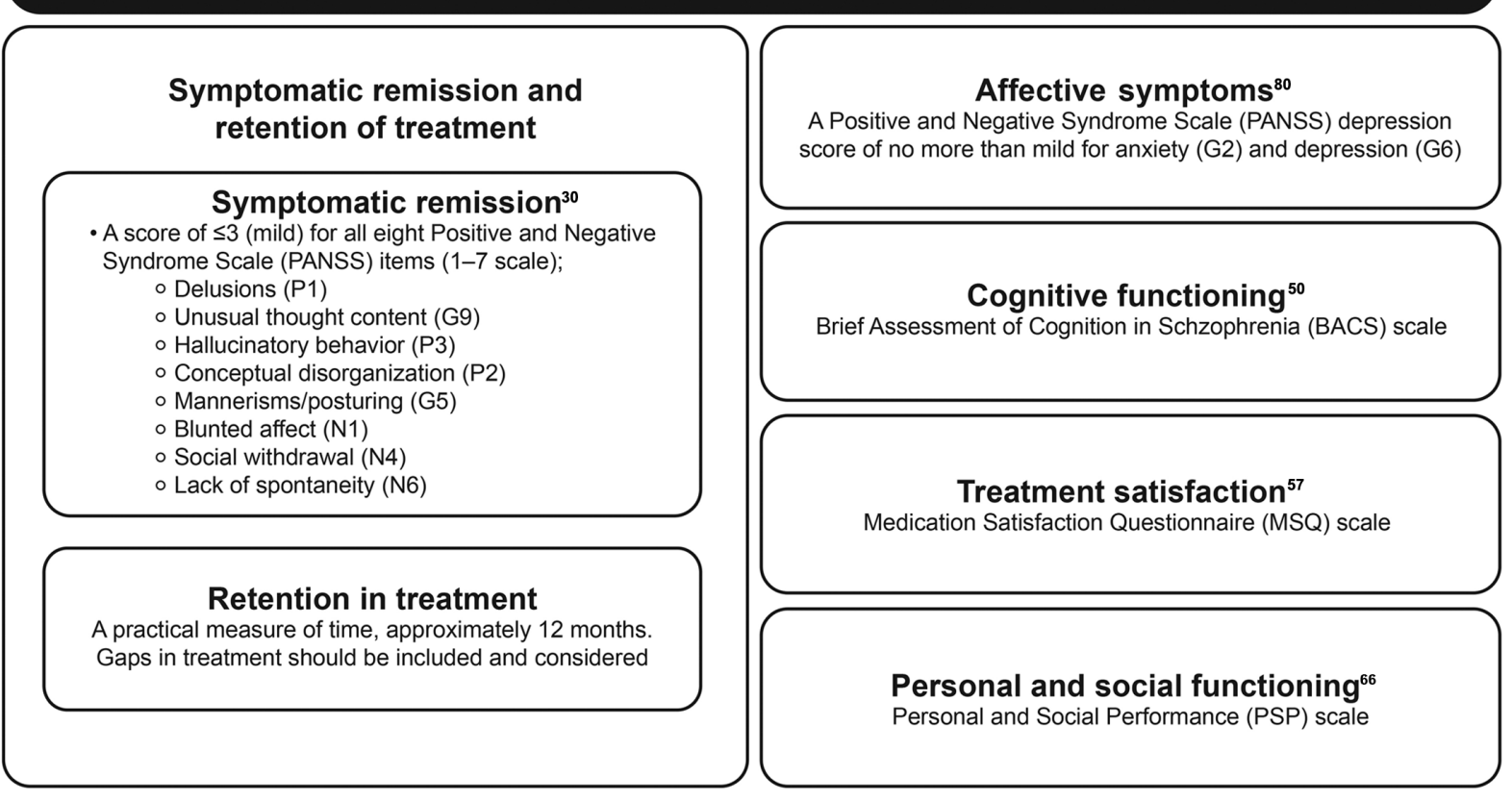

Figure 2 Recommended domains and scales that make up the framework to assess the effectiveness of antipsychotics in schizophrenia. For symptomatic remission, items in parentheses refer to the specific PANSS items 
Table 3 Summary of proposed assessments to enhance the measurement of treatment effectiveness in patients with schizophrenia

\begin{tabular}{lllll}
\hline Domain & Instrument & $\begin{array}{l}\text { Number of } \\
\text { items }\end{array}$ & Time required & $\begin{array}{l}\text { Frequency of } \\
\text { assessment }\end{array}$ \\
\hline Symptomatic remission & PANSS & 8 items & 16 minutes & Monthly \\
Retention in treatment & - & - & - & Yearly \\
Affective symptoms & PANSS & 2 items & 4 minutes & Monthly \\
Cognitive functioning & BACS & 6 tests & 35 minutes & Yearly \\
Treatment satisfaction & MSQ & 1 item & 2 minutes & Monthly \\
Personal and social functioning & PSP & 4 domains & 10 minutes & Monthly \\
\hline
\end{tabular}

Abbreviations: BACS, Brief Assessment of Cognition in Schizophrenia; MSQ, Medication Satisfaction Questionnaire; PANSS, Positive and Negative Syndrome Scale; PSP, Personal and Social Performance.

\section{Conclusion}

Effectiveness in schizophrenia is poorly defined, with numerous different effectiveness outcomes utilized in clinical studies. To aid clinical practice, particularly in the assessment of treatment effectiveness in patients with schizophrenia, a concise and easy-to-use framework is required. European psychiatrists and psychopharmacologists convened to develop a proposal for the framework of treatment effectiveness in schizophrenia. This framework consists of five domains: 1) symptomatic remission and retention of treatment; 2) affective symptoms; 3) cognitive functioning; 4) treatment satisfaction; and 5) personal and social functioning. The aim of the proposal is to promote a patient-centered approach to the assessment of treatment effectiveness in patients with schizophrenia, and to provide clinically applicable scales for each domain that are appropriate for use in everyday practice.

It is anticipated that an increased awareness of the five domains presented here will improve clinical practice, especially following discharge of patients from hospital into the community setting; stimulate greater investigation into often overlooked aspects, such as cognitive functioning and affective symptoms; and promote development of better assessment tools that are designed for clinical rather than academic use.

\section{Acknowledgments}

Medical writing and editorial assistance in the preparation of this manuscript was provided by apothecom scopemedical ltd, Sevenoaks, UK, and was supported by Janssen. The authors have not received payment from Janssen for their involvement in preparing this article. The authors would like to acknowledge the contribution made by the attendees of the expert meetings and large educational meeting that helped to shape the content of this publication. The authors would also like to acknowledge the significant contributions Professor René S Kahn, Professor Tom Burns, Professor Bill Deakin, and Professor Gavin Reynolds made to the discussions and development of the framework presented here.

\section{Disclosure}

Authors with a research interest in treatment effectiveness were identified by Janssen. All authors with the exception of Luca Pani were remunerated for their attendance at meetings where the content of this paper was discussed. Luca Pani is currently the General Director of the Italian Drug Agency (AIFA) and a Member of the Committee for Human Medicinal Products (CHMP) at the European Medicines Agency (EMA). According to EMA/513078/2010 policy 0044 on the handling of conflicts of interests of Scientific Committee members and experts, the views expressed in this paper are personal and may not be understood or quoted as being made on behalf of or reflecting the position of the EMA or one of its committees or working parties. Georg Juckel has received honoraria as an educational speaker sponsored by AstraZeneca, Janssen, Eli Lilly, Bristol-Myers Squibb, Pfizer, and Servier. He received grants for investigator initiated trails from Janssen, Astra-Zeneca, Lilly, and BMS. Andrea de Bartolomeis has received honoraria as an educational speaker sponsored by Astra-Zeneca Italia, Janssen-Cilag, Eli Lilly, Bristol-Myers Squibb, and Lundbeck, as well as unrestricted grants for research activity at the Laboratory of Molecular Psychiatry, Department of Neuroscience, University of Napoli Federico II from Astra-Zeneca, Janssen, and Lundbeck. Philip Gorwood has received grants from Eli Lilly and Servier and honoraria (advisory boards and presentations in congresses) from Bristol-Myers Squibb, Janssen, Lundbeck, Servier, and Wyeth. He has a paid position from the University of Paris-Descartes and Hospital Sainte-Anne (Paris). Sergey Mosolov, Julio Sanjuan, and Alessandro Rossi report no conflicts of interest in this work.

\section{References}

1. American Psychiatric Association. Practice guideline for the treatment of patients with schizophrenia. 2nd ed. 2004.

2. Falkai P, Wobrock T, Lieberman J, et al. World Federation of Societies of Biological Psychiatry (WFSBP) guidelines for biological treatment of schizophrenia, Part 1: acute treatment of schizophrenia. World J Biol Psychiatry. 2005;6(3):132-191. 
3. Falkai P, Wobrock T, Lieberman J, et al. World Federation of Societies of Biological Psychiatry (WFSBP) guidelines for biological treatment of schizophrenia, part 2: long-term treatment of schizophrenia. World J Biol Psychiatry. 2006;7(1):5-40.

4. Correll CU, Leucht S, Kane JM. Lower risk for tardive dyskinesia associated with second-generation antipsychotics: a systematic review of 1-year studies. Am J Psychiatry. 2004;161(3):414-425.

5. Heal DJ, Gosden J, Jackson HC, Cheetham SC, Smith SL. Metabolic consequences of antipsychotic therapy: preclinical and clinical perspectives on diabetes, diabetic ketoacidosis, and obesity. Handb Exp Pharmacol. 2012;(212):135-164.

6. Leucht S, Corves C, Arbter D, Engel RR, Li C, Davis JM. Secondgeneration versus first-generation antipsychotic drugs for schizophrenia: a meta-analysis. Lancet. 2009;373(9657):31-41.

7. Leucht S, Komossa K, Rummel-Kluge C, et al. A meta-analysis of head-to-head comparisons of second-generation antipsychotics in the treatment of schizophrenia. Am J Psychiatry. 2009;166(2):152-163.

8. Richelson E, Souder T. Binding of antipsychotic drugs to human brain receptors focus on newer generation compounds. Life Sci. 2000;68(1): 29-39.

9. Nasrallah HA. Atypical antipsychotic-induced metabolic side effects: insights from receptor-binding profiles. Mol Psychiatry. 2008;13(1): $27-35$.

10. Johnsen E, Jørgensen HA. Effectiveness of second generation antipsychotics: a systematic review of randomized trials. BMC Psychiatry. 2008;8:31.

11. Kahn RS, Fleischhacker WW, Boter H, et al. Effectiveness of antipsychotic drugs in first-episode schizophrenia and schizophreniform disorder: an open randomised clinical trial. Lancet. 2008;371(9618): 1085-1097.

12. Lieberman JA, Stroup TS, McEvoy JP, et al. Effectiveness of antipsychotic drugs in patients with chronic schizophrenia. $N$ Engl J Med. 2005;353(12):1209-1223.

13. Zimbroff DL. Management of acute psychosis: from emergency to stabilization. CNS Spectr. 2003;8(11 Suppl 2):10-15.

14. Casey DE. Long-term treatment goals: enhancing healthy outcomes. CNS Spectr. 2003;8(11 Suppl 2):26-28.

15. Keshavan MS, Nasrallah HA, Tandon R. Schizophrenia, "Just the Facts" 6. Moving ahead with the schizophrenia concept: from the elephant to the mouse. Schizophr Res. 2011;127(1-3):3-13.

16. Stroup TS, McEvoy JP, Swartz MS, et al. The National Institute of Mental Health Clinical Antipsychotic Trials of Intervention Effectiveness (CATIE) project: schizophrenia trial design and protocol development. Schizophr Bull. 2003;29(1):15-31.

17. Dossenbach M, Erol A, el Mahfoud KM, et al. Effectiveness of antipsychotic treatments for schizophrenia: interim 6-month analysis from a prospective observational study (IC-SOHO) comparing olanzapine, quetiapine, risperidone, and haloperidol. J Clin Psychiatry. 2004;65(3): $312-321$.

18. Haro JM, Edgell ET, Novick D, et al. Effectiveness of antipsychotic treatment for schizophrenia: 6-month results of the Pan-European Schizophrenia Outpatient Health Outcomes (SOHO) study. Acta Psychiatr Scand. 2005;111(3):220-231

19. Jones PB, Barnes TR, Davies L, et al. Randomized controlled trial of the effect on Quality of Life of second- vs first-generation antipsychotic drugs in schizophrenia: Cost Utility of the Latest Antipsychotic Drugs in Schizophrenia Study (CUtLASS 1). Arch Gen Psychiatry. 2006;63(10): 1079-1087.

20. Lewis SW, Barnes TR, Davies L, et al. Randomized controlled trial of effect of prescription of clozapine versus other second-generation antipsychotic drugs in resistant schizophrenia. Schizophr Bull. 2006;32(4): 715-723.

21. Tandon R, Marcus RN, Stock EG, et al. A prospective, multicenter, randomized, parallel-group, open-label study of aripiprazole in the management of patients with schizophrenia or schizoaffective disorder in general psychiatric practice: Broad Effectiveness Trial With Aripiprazole (BETA). Schizophr Res. 2006;84(1):77-89.
22. McEvoy JP, Lieberman JA, Perkins DO, et al. Efficacy and tolerability of olanzapine, quetiapine, and risperidone in the treatment of early psychosis: a randomized, double-blind 52-week comparison. Am J Psychiatry. 2007;164(7):1050-1060.

23. Perkins DO, Gu H, Weiden PJ, McEvoy JP, Hamer RM, Lieberman JA. Predictors of treatment discontinuation and medication nonadherence in patients recovering from a first episode of schizophrenia, schizophreniform disorder, or schizoaffective disorder: a randomized, double-blind, flexible-dose, multicenter study. J Clin Psychiatry. 2008; 69(1):106-113.

24. Nasrallah HA, Targum SD, Tandon R, McCombs JS, Ross R. Defining and measuring clinical effectiveness in the treatment of schizophrenia. Psychiatr Serv. 2005;56(3):273-282.

25. Kinon BJ, Chen L, Ascher-Svanum H, et al. Challenging the assumption that improvement in functional outcomes is delayed relative to improvement in symptoms in the treatment of schizophrenia. Schizophr Res. 2010;118(1-3):176-182.

26. Stauffer VL, Case M, Kinon BJ, et al. Early response to antipsychotic therapy as a clinical marker of subsequent response in the treatment of patients with first-episode psychosis. Psychiatry Res. 2011;187(1-2): $42-48$.

27. Giegling I, Porcelli S, Balzarro B, et al. Antipsychotic response in the first week predicts later efficacy. Neuropsychobiology. 2012;66(2):100-105.

28. Ascher-Svanum H, Zhu B, Faries DE, Lacro JP, Dolder CR, Peng X. Adherence and persistence to typical and atypical antipsychotics in the naturalistic treatment of patients with schizophrenia. Patient Prefer Adherence. 2008:2:67-77.

29. Liu-Seifert H, Adams DH, Kinon BJ. Discontinuation of treatment of schizophrenic patients is driven by poor symptom response: a pooled post-hoc analysis of four atypical antipsychotic drugs. BMC Med. 2005;3:21.

30. Andreasen NC, Carpenter WT Jr, Kane JM, Lasser RA, Marder SR, Weinberger DR. Remission in schizophrenia: proposed criteria and rationale for consensus. Am J Psychiatry. 2005;162(3):441-449.

31. Emsley R, Oosthuizen P, Koen L, Niehaus DJ, Medori R, Rabinowitz J. Remission in patients with first-episode schizophrenia receiving assured antipsychotic medication: a study with risperidone long-acting injection. Int Clin Psychopharmacol. 2008;23(6):325-331.

32. Lauriello J, Lambert T, Anderson S, Lin D, Taylor CC, McDonnell D. An 8-week, double-blind, randomized, placebo-controlled study of olanzapine long-acting injection in acutely ill patients with schizophrenia. J Clin Psychiatry. 2008;69:790-799.

33. Kane JM, Sanchez R, Perry PP, et al. Aripiprazole intramuscular depot as maintenance treatment in patients with schizophrenia: a 52-week, multicenter, randomized, double-blind, placebo-controlled study. JClin Psychiatry. 2012;73:617-624.

34. Potkin SG, Weiden PJ, Loebel AD, Warrington LE, Watsky EJ, Siu CO. Remission in schizophrenia: 196-week, double-blind treatment with ziprasidone vs haloperidol. Int J Neuropsychopharmacol. 2009;12(9): $1233-1248$

35. Gorwood P, Peuskens J; European Group On Functional Outcomes, Remission in Schizophrenia. Setting new standards in schizophrenia outcomes: Symptomatic remission 3 years before versus after the andreasen criteria. Eur Psychiatry. 2012;27(3):170-175.

36. Lambert M, Karow A, Leucht S, Schimmelmann BG, Naber D. Remission in schizophrenia: validity, frequency, predictors, and patients' perspective 5 years later. Dialogues Clin Neurosci. 2010;12(3): 393-407.

37. AlAqeel B, Margolese HC. Remission in schizophrenia: critical and systematic review. Harv Rev Psychiatry. 2012;20:281-297.

38. Hamilton M. A rating scale for depression. J Neurol Neurosurg Psychiatry. 1960;23:56-62.

39. Hamilton M. Development of a rating scale for primary depressive illness. Br J Soc Clin Psychol. 1967;6(4):278-296.

40. Montgomery SA, Asberg M. A new depression scale designed to be sensitive to change. Br J Psychiatry. 1979;134:382-389. 
41. Addington D, Addington J, Maticka-Tyndale E. Assessing depression in schizophrenia: the Calgary Depression Scale. Br J Psychiatry Suppl. 1993;(22):39-44.

42. Hofer A, Rettenbacher MA, Edlinger M, et al. Outcomes in schizophrenia outpatients treated with amisulpride or olanzapine. Pharmacopsychiatry. 2007;40(1):1-8.

43. Kahn RS, Schulz SC, Palazov VD, et al. Efficacy and tolerability of once-daily extended release quetiapine fumarate in acute schizophrenia: a randomized, double-blind, placebo-controlled study. J Clin Psychiatry. 2007;68(6):832-842.

44. Meltzer HY, Bobo WV, Nuamah IF, et al. Efficacy and tolerability of oral paliperidone extended-release tablets in the treatment of acute schizophrenia: pooled data from three 6-week, placebo-controlled studies. J Clin Psychiatry. 2008;69(5):817-829.

45. Keefe RS, Bilder RM, Davis SM, et al. Neurocognitive effects of antipsychotic medications in patients with chronic schizophrenia in the CATIE Trial. Arch Gen Psychiatry. 2007;64(6): 633-647.

46. Keefe RS, Poe M, Walker TM, Kang JW, Harvey PD. The Schizophrenia Cognition Rating Scale: an interview-based assessment and its relationship to cognition, real-world functioning, and functional capacity. Am $J$ Psychiatry. 2006;163(3):426-432.

47. Galderisi S, Davidson M, Kahn RS, et al. Correlates of cognitive impairment in first episode schizophrenia: the EUFEST study. Schizophr Res. 2009;115(2-3):104-114.

48. Mausbach BT, Depp CA, Bowie CR, et al. Sensitivity and specificity of the UCSD Performance-based Skills Assessment (UPSA-B) for identifying functional milestones in schizophrenia. Schizophr Res. 2011;132(2-3):165-170.

49. Nuechterlein KH, Green MF, Kern RS, et al. The MATRICS Consensus Cognitive Battery, part 1: test selection, reliability, and validity. Am J Psychiatry. 2008;165(2):203-213.

50. Keefe RS, Goldberg TE, Harvey PD, Gold JM, Poe MP, Coughenour L. The Brief Assessment of Cognition in Schizophrenia: reliability, sensitivity, and comparison with a standard neurocognitive battery. Schizophr Res. 2004;68(2-3):283-297.

51. Rummel-Kluge C, Kissling W. Psychoeducation for patients with schizophrenia and their families. Expert Rev Neurother. 2008;8(7) 1067-1077.

52. Larsen DL, Attkisson CC, Hargreaves WA, Nguyen TD. Assessment of client/patient satisfaction: development of a general scale. Eval Program Plann. 1979;2(3):197-207.

53. Ruggeri M, Lasalvia A, Dall'Agnola R, et al. Development, internal consistency and reliability of the Verona Service Satisfaction Scale European Version. EPSILON Study 7. European Psychiatric Services: Inputs Linked to Outcome Domains and Needs. Br J Psychiatry Suppl. 2000;(39):s41-s48.

54. Richardson M, Katsakou C, Torres-Gonzalez F, Onchev G, Kallert T, Priebe S. Factorial validity and measurement equivalence of the Client Assessment of Treatment Scale for psychiatric inpatient care a study in three European countries. Psychiatry Res. 2011;188(1): 156-160.

55. Endicott J, Nee J, Harrison W, Blumenthal R. Quality of Life Enjoyment and Satisfaction Questionnaire: a new measure. Psychopharmacol Bull. 1993;29(2):321-326.

56. Atkinson MJ, Sinha A, Hass SL, et al. Validation of a general measure of treatment satisfaction, the Treatment Satisfaction Questionnaire for Medication (TSQM), using a national panel study of chronic disease. Health Qual Life Outcomes. 2004;2:12.

57. Gharabawi GM, Greenspan A, Rupnow MF, et al. Reduction in psychotic symptoms as a predictor of patient satisfaction with antipsychotic medication in schizophrenia: data from a randomized double-blind trial. BMC Psychiatry. 2006;6:45.

58. Vernon MK, Revicki DA, Awad AG, et al. Psychometric evaluation of the Medication Satisfaction Questionnaire (MSQ) to assess satisfaction with antipsychotic medication among schizophrenia patients. Schizophr Res. 2010;118(1-3):271-278.
59. Juckel G, Morosini PL. The new approach: psychosocial functioning as a necessary outcome criterion for therapeutic success in schizophrenia. Curr Opin Psychiatry. 2008;21(6):630-639.

60. Galderisi S, Mucci A, Bitter I, et al. Persistent negative symptoms in first episode patients with schizophrenia: results from the European First Episode Schizophrenia Trial. Eur Neuropsychopharmacol. 2013;23: 196-204.

61. Morgan C, Burns T, Fitzpatrick R, Pinfold V, Priebe S. Social exclusion and mental health: conceptual and methodological review. $\mathrm{Br} \mathrm{J}$ Psychiatry. 2007;191:477-483.

62. Patrick DL, Burns T, Morosini P, Gagnon DD, Rothman M, Adriaenssen I. Measuring social functioning with the personal and social performance scale in patients with acute symptoms of schizophrenia: interpretation of results of a pooled analysis of three Phase III trials of paliperidone extended-release tablets. Clin Ther. 2010;32(2): 275-292.

63. Mosolov SN, Potapov AV, Ushakov UV. Remission in schizophrenia: results of cross-sectional with 6-month follow-up period and 1-year observational therapeutic studies in an outpatient population. Ann Gen Psychiatry. 2012;11:1.

64. Mosolov SN, Potapov AV, Ushakov UV, Shararenko AA, Kostyukova AB. Design and validation of standardized clinical and functional remission criteria in schizophrenia. Neuropsychiatr Dis Treat. 2014;10: 167-181.

65. Karow A, Naber D, Lambert M, Moritz S; EGOFORS initiative. Remission as perceived by people with schizophrenia, family members and psychiatrists. Eur Psychiatry. 2012;27(6):426-431.

66. Morosini PL, Magliano L, Brambilla L, Ugolini S, Pioli R. Development, reliability and acceptability of a new version of the DSM-IV Social and Occupational Functioning Assessment Scale (SOFAS) to assess routine social functioning. Acta Psychiatr Scand. 2000;101(4): 323-329.

67. Nafees B, van Hanswijck de Jonge P, Stull D, et al. Reliability and validity of the Personal and Social Performance scale in patients with schizophrenia. Schizophr Res. 2012;140(1-3):71-76.

68. Brissos S, Palhava F, Marques JG, et al. The Portuguese version of the Personal and Social Performance Scale (PSP): reliability, validity, and relationship with cognitive measures in hospitalized and community schizophrenia patients. Soc Psychiatry Psychiatr Epidemiol. 2012; 47(7): 1077-1086.

69. Tianmei S, Liang S, Yun'ai S, et al. The Chinese version of the Personal and Social Performance Scale (PSP): validity and reliability. Psychiatry Res. 2011;185(1-2):275-279.

70. Apiquian R, Elena UR, Herrera-Estrella M, et al. Validity of the Spanish version of the Personal and Social Performance scale in schizophrenia. Schizophr Res. 2009;112(1-3):181-186.

71. Nasrallah H, Morosini P, Gagnon DD. Reliability, validity and ability to detect change of the Personal and Social Performance scale in patients with stable schizophrenia. Psychiatry Res. 2008;161(2):213-224.

72. Patrick DL, Burns T, Morosini P, et al. Reliability, validity and ability to detect change of the clinician-rated Personal and Social Performance scale in patients with acute symptoms of schizophrenia. Curr Med Res Opin. 2009;25(2):325-338.

73. Figueira ML, Brissos S. Measuring psychosocial outcomes in schizophrenia patients. Curr Opin Psychiatry. 2011;24:91-99.

74. Mendes Menzes AK, Macedo G, Mattos P, Reis de Sá Júnior A, Louza MR. Personal and Social Performance (PSP) scale for patients with schizophrenia: translation to Portuguese, cross-cultural adaption and interrater reliability. J Bras Psiquiatr. 2012;61:176-180.

75. Juckel G, Schaub D, Fuchs N, et al. Validation of the Personal and Social Performance (PSP) Scale in a German sample of acutely ill patients with schizophrenia. Schizophr Res. 2008;104(1-3): 287-293.

76. Srisurapont M, Arunpongpaisal S, Chuntaruchikapong S, et al. Cross-cultural validation and inter-rater reliability of the Personal and Social Performance Scale, Thai Version. J Med Assoc Thai. 2008;91: 1603-1608. 
77. Burns T, Patrick D. Social functioning as an outcome measure in schizophrenia studies. Acta Psychiatr Scand. 2007;116(6):403-418.

78. Schaub D, Brüne M, Bierhoff HW, Juckel G. Comparison of self- and clinician's ratings of Personal and Social Performance in patients with schizophrenia: the role of insight. Psychopathology. 2012;45(2): 109-116.
79. Schaub D, Brüne M, Jaspen E, Pajonk FG, Bierhoff HW, Juckel G. The illness and everyday living: close interplay of psychopathological syndromes and psychosocial functioning in chronic schizophrenia. Eur Arch Psychiatry Clin Neurosci. 2011;261(2):85-93.

80. Kay SR, Fiszbein A, Opler LA. The positive and negative syndrome scale (PANSS) for schizophrenia. Schizophr Bull. 1987;13(2):261-276.

\section{Publish your work in this journal}

Neuropsychiatric Disease and Treatment is an international, peerreviewed journal of clinical therapeutics and pharmacology focusing on concise rapid reporting of clinical or pre-clinical studies on a range of neuropsychiatric and neurological disorders. This journal is indexed on PubMed Central, the 'PsycINFO' database and CAS, and is the official journal of The International Neuropsychiatric Association (INA). The manuscript management system is completely online and includes a very quick and fair peer-review system, which is all easy to use. Visit http://www.dovepress.com/testimonials.php to read real quotes from published authors.

Submit your manuscript here: http://www.dovepress.com/neuropsychiatric-disease-and-treatment-journal 